\title{
Effects of lid debris debridement combined with meibomian gland expression on the ocular surface MMP-9 levels and clinical outcomes in moderate and severe meibomian gland dysfunction
}

Su Young Moon ${ }^{\dagger}$, Sol Ah Han ${ }^{\dagger}$, Hye Ji Kwon, So Young Park, Jae Hyuck Lee, Ho Seok Chung, Jae Yong Kim, Hungwon Tchah and Hun Lee ${ }^{*}$ (D)

\begin{abstract}
Background: To evaluate the effects of lid debris debridement and meibomian gland expression (MGX) on extracellular matrix metalloproteinase-9 (MMP-9) levels and clinical outcomes of moderate and severe MGD.

Methods: In this retrospective case series study, a total 48 eyes of 24 patients with moderate and severe MGD underwent one session of lid debris debridement using the BlephEx combined with MGX. We evaluated the tear film break-up time (TBUT), corneal and conjunctival fluorescein staining scores, Schirmer 1 test, biomicroscopic examination of lid margins and meibomian gland (MG), ocular surface disease index (OSDI) questionnaire score, and extracellular MMP-9 levels using a point-of-care MMP-9 immunoassay device before and 4 weeks after lid debris debridement and MGX. Linear mixed model and generalized estimating equations model were used to evaluate possible differences.

Results: There were significant improvements in the TBUT $(P=0.002)$, SICCA and Oxford staining scores (all $P<$ 0.001), lid margin telangiectasia ( $P<0.001$ for upper and lower eyelids), lid thickness $(P<0.001$ for upper and lower eyelids), MG orifice plugging ( $P<0.001$ for upper and lower eyelids), meibum color $(P=0.026$ for upper eyelid, $P<$ 0.001 for lower eyelid), meibum consistency $(P<0.001$ for upper and lower eyelids), meibum grade $(P<0.001)$, MGD stage $(P<0.001)$, and OSDI score $(P=0.002)$. MMP-9 immunoassay positivity rate significantly decreased from 83.3 to $50.0 \% 4$ weeks after treatment $(P=0.014)$.

(Continued on next page)
\end{abstract}

\footnotetext{
* Correspondence: yhun777@amc.seoul.kr; yhun777@hanmail.net

†Su Young Moon and Sol Ah Han contributed equally to this work.

Department of Ophthalmology, Asan Medical Center, University of Ulsan

College of Medicine, 88 Olympic-Ro 43-Gil, Songpa-Gu, Seoul 05505, South

Korea
}

(C) The Author(s). 2021 Open Access This article is licensed under a Creative Commons Attribution 4.0 International License, which permits use, sharing, adaptation, distribution and reproduction in any medium or format, as long as you give appropriate credit to the original author(s) and the source, provide a link to the Creative Commons licence, and indicate if changes were made. The images or other third party material in this article are included in the article's Creative Commons licence, unless indicated otherwise in a credit line to the material. If material is not included in the article's Creative Commons licence and your intended use is not permitted by statutory regulation or exceeds the permitted use, you will need to obtain permission directly from the copyright holder. To view a copy of this licence, visit http://creativecommons.org/licenses/by/4.0/ The Creative Commons Public Domain Dedication waiver (http://creativecommons.org/publicdomain/zero/1.0/) applies to the data made available in this article, unless otherwise stated in a credit line to the data. 
(Continued from previous page)

Conclusions: In patients with moderate to severe MGD, lid debris debridement using the BlephEx combined with MGX improved clinical findings, subjective symptoms, meibomian gland function, along with ocular surface MMP-9 level. We hereby suggest lid debris debridement using BlephEx combined with MGX as an effective clinical strategy for treatment of moderate to severe MGD.

Keywords: Dry eye, Lid debris debridement, Matrix metalloproteinase-9, Matrix metalloproteinase-9 immunoassay device, Meibomian gland dysfunction, Meibomian gland expression

\section{Background}

Dry eye disease, despite its frequent diagnosis in ophthalmology, is often intractable despite diverse treatment efforts of ophthalmologists. Patients report various ocular symptoms caused by tear film instability, hyperosmolarity, ocular surface inflammation, and neurosensory abnormalities [1]. Meibomian gland dysfunction (MGD) is a prevalent condition and a major cause of dry eye [2]. MGD is commonly characterized by terminal duct obstruction and changes in lipid-based glandular secretion, resulting in a deficient outer protective layer of the tear film, and is generally associated with a chronic course with intermittent flares [3-5].

The goal of MGD treatment is to provide long-term alleviation of the symptoms for patients by improving the quality of the meibum and tear film stability, and by decreasing ocular surface inflammation. Conventional eyelid management is not sufficient to resolve inspissated meibum [6]. Thus, additional measures are needed to relieve obstructed meibomian gland efficiently and to modulate subsequent inflammatory processes in moderate and severe MGD [7, 8]. Systemic tetracycline, doxycycline, minocycline, and topical ophthalmic solutions, such as azithromycin, loteprednol etabonate, cyclosporine, and diquafosol, can be effective when prescribed concurrently with conventional eyelid management [7-11]. Recently, several studies have reported the efficacy of intensive pulsed light (IPL) treatment for MGD, even for the refractory type [11-17].

Lid debris debridement using the BlephEx system (RySurg, Fort Worth, FL, USA) is a novel form of eyelid management that removes the accumulated bacterial biofilm from the lid margin and scurf from the eyelashes [18]. However, to the best of our knowledge, previous studies have not evaluated the effect of mechanical lid debridement on ocular surface inflammation using the matrix metalloproteinase-9 (MMP-9) immunoassay device (RPS InflammaDry; Rapid Pathogen Screening Inc., Sarasota, FL, USA) to the assess extracellular MMP-9. Therefore, this study aims to investigate the effect of lid debris debridement by measuring the extracellular MMP-9 levels using an MMP-9 immunoassay device and by evaluating the change in clinical findings, subjective symptoms, and meibomian gland function in patients with moderate to severe MGD.

\section{Methods}

This study was approved by the Institutional Review Board of the Asan Medical Center and the University of Ulsan College of Medicine, Seoul, South Korea (20200096) and performed in accordance with the Declaration of Helsinki and relevant guidelines.

Patients diagnosed with moderate and severe MGD at Asan Medical Center IPL Dry Eye Clinic who met the inclusion criteria were enrolled in the study. Patients had to be at least 20 years old. Moderate and severe MGD was diagnosed based on tarsal conjunctival erythema, bulbar conjunctival hyperemia, telangiectasia, thickening and irregularity of the eyelid margin, and meibomian gland orifice inclusions. MGD staging was determined using the criteria stated in the International Workshop on Meibomian Gland Dysfunction described by the Tear Film and Ocular Surface Society. Patients with moderate degree of symptoms including ocular discomfort, itching or photophobia, moderate degree of clinical signs (plugging, telangiectasia, moderately altered secretions from grade 8 to less than 13, expressibility 2), and mild to moderate conjunctival and peripheral corneal staining were diagnosed with moderate MGD. Diagnosis of severe MGD was made according to following criteria: patient report of considerable ocular discomfort, itching or photophobia, officebased clinical assessment (dropout, displacement, severely altered secretions of grade 13 or greater, expressibility 3), notable degree of conjunctival and corneal staining or clinical indications of elevated inflammation, including but not limited to moderate conjunctival hyperemia, phlyctenules $[19,20]$. The exclusion criteria are as follows: history of previous ocular surgery, diagnosis of glaucoma or ocular hypertension, clinical sign of active ocular infection or non-dry eye ocular inflammation, ocular allergy, diagnosis of autoimmune disease, use of contact lenses during the study period, presence of punctal occlusion, use of anti-inflammatory eye-drops and oral antibiotics within a month before enrollment, active skin lesions or previously diagnosed skin pathology, ocular rosacea, pregnancy or lactation.

\section{Measurements}

Clinical evaluation was performed before initiating the treatment and 4 weeks after the treatment. The 
parameters used for clinical examination include extracellular MMP-9 levels using the MMP-9 immunoassay device, tear film break-up time (TBUT), corneal and conjunctival staining score (Sjögren's International Collaborative Clinical Alliance [SICCA] ocular staining and Oxford staining score), Schirmer 1 test performed without topical anesthetics, lid margin abnormalities and meibum grade [21-24].

The MMP-9 level of the ocular surface was measured using the MMP-9 immunoassay device, which is a pointof-care test that analyzes the tear samples collected in the office [25]. Existence of one blue line and one red line in the MMP-9 test result window means a positive test result (MMP-9 $\geq 40 \mathrm{ng} / \mathrm{mL}$ : strong positive, positive, and weak positive), while single blue line means a negative test result (MMP-9 < $40 \mathrm{ng} / \mathrm{mL}$ : trace and negative) [24]. We also measured the MMP-9 level based on the change in the intensity of color at $10 \mathrm{~min}$ using standardized photographs as a reference on a scale of 0 to 4: 0 , none; 1 , trace; 2 , weak positive; 3 , positive; and 4 , strong positive [24].

A single sterile sodium fluorescein strip was wet with non-preserved saline solution then touched to the bulbar conjunctiva to measure TBUT. TBUT was defined as the time lapse between a blink and appearance of the first spot on the corneal surface and the average of 3 measurements was taken. Corneal and conjunctival fluorescein staining was examined, which was followed by Schirmer 1 test (without topical anesthetic). Fluorescein staining was graded according to the SICCA and Oxford scale.

The lid margin was examined under slit-lamp microscope and recorded separately for upper and lower lids. We assessed telangiectasia (on a scale of $0-3$, with $0=$ no findings; 1 = mild telangiectasia $2=$ moderate telangiectasia or redness; and $3=$ severe telangiectasia or redness), anterior or posterior replacement of the mucocutaneous junction, and lid abnormalities, including irregularity, thickness, and plugging (on a scale of 0 2 , with $0=$ no finding; $1=$ mild; and $2=$ severe) [21-23]. To assess the meibum grade, the degree of digital pressure applied on the upper tarsus during MG expression was recorded by the physician, the grading was as follows: grade $0=$ clear meibum easily expressed; grade $1=$ cloudy meibum expressed with mild pressure; grade $2=$ cloudy meibum expressed with more than moderate pressure; and grade $3=$ no meibum expression, even with firm pressure [26]. The color (on a scale of 1-3, $1=$ clear; 2 =cloudy; and $3=$ yellow) and consistency of expressed meibum (on a scale of $1-3,1=$ oily; $2=$ creamy; and 3 =toothpaste like) were assessed for both upper and lower eyelids using the meibomian gland expressor forceps [21].

Patients self-reported their symptoms using the ocular surface disease index (OSDI) questionnaire and ocular irritation symptom (ocular discomfort, itching, and photophobia with limitations of activities) and the severity was graded on a scale of 0 (no symptom) to 3 (severe symptom). In order to minimize its effect on each other, the order of clinical exams was identical for all patients, starting with the MMP-9 immunoassay which was followed by Schirmer 1 test, biomicroscopic examination of the TBUT, corneal and conjunctival fluorescein staining, and lastly examination of the lid margins and meibomian glands [24]. After completing the series of physical exams, patients filled out the OSDI questionnaire and ocular irritation symptom score.

\section{Lid debris debridement combined with meibomian gland expression (MGX)}

Patients underwent one session of BlephEx treatment with MGX performed by a single physician (HL). For any patients already in use of topical or systemic medication for dry eye disease, a minimum of 4 week washout period was required before enrolling the study. The BlephEx system was designed to exfoliate the eyelid margin with a rapidly spinning sponge-tipped microbrush and a foam cleanser that is swept back and forth along the lid margin for approximately 1-2 min to remove the bacterial biofilm, lid debris, and Demodex folliculorum mites $[18,27,28]$. The procedure is completed with 4 disposable brushes ( 1 for each eyelid margin) after removal of all visible lid scurf and cylindrical dandruff. Immediately after BlephEx treatment, meibomian gland of both upper and lower eyelids of each eye was expressed using a meibomian gland forcep after administering topical 0.4\% oxybuprocaine hydrochloride as anesthetics. Daily lid hygiene regimen using an eyelid scrub and warm compression was recommended to all patients in addition to use of $0.18 \%$ sodium hyaluronate (Kynex2, Alcon Laboratory, Seoul, Korea) on demand during the follow-up period.

\section{Statistical analysis}

Data were analyzed using linear mixed model, with AR (1) correlation structure. Generalized estimating equations model was used to compare non-continuous variables including ocular irritation symptom score, mucocutaneous junction replacement, meibum grade, and MGD stage. Linear mixed model and $x^{2}$ test were used to analyze MMP-9 immunoassay results. Statistical analysis was performed using SPSS software version 25.0 (IBM, Armonk, NY, USA). A $P$ value of less than 0.05 was considered statistically significant.

\section{Results}

The mean age of the patients was $63.1 \pm 10.6$ (range, 4686) years. The majority of the subjects was women (71\%). Table 1 shows the changes in the clinical findings 
and symptoms before and at 4 weeks after lid debris debridement and MGX. A significant increase in the TBUT $(P=0.002)$, along with improvement in SICCA ocular staining score $(P<0.001)$ and Oxford staining score $(P<0.001)$ were observed at 4 weeks after the treatment. However, there was no significant difference in the Schirmer test results $(P=0.703)$. Significant improvement in mean OSDI score $(P=0.002)$ and ocular irritation symptom score $(P<0.001)$ indicates that there is a notable alleviation of symptoms. Significant improvement in lid margin abnormality, including telangiectasia, plugging, and thickness was detected at 4 weeks after the treatment (Table 2). There was a significant decrease in the lid margin telangiectasia, plugging, and thickness scores in both the upper and lower eyelids (all $P<0.001)$. However, we did not observe a significant change in the lid margin irregularity score and mucocutaneous junction replacement.

Notable improvements in the meibomian gland function in both the upper and lower eyelids were observed in terms of meibum color $(P=0.026$ for the upper eyelid and $P<0.001$ for the lower eyelid) and meibum consistency $(P<0.001$ for the upper and lower eyelids) (Table 2). Accordingly, the proportion of eyes of MGD grade 2 or 3 decreased significantly $(P<0.001)$. Finally, the proportion of eyes with MGD stage 3 or 4 showed a significant reduction $(P<0.001)$ (Fig. 1$)$.

Significant improvement in the mean ocular surface MMP-9 level $(P=0.029)$ was observed measured based on a scale of 0 to 4 for intensity of color $(0$, none; 1 , trace; 2 , weak; 3 , positive; and 4 , strong positive) (Table 3). Changes in MMP-9 level before and after eyelid debris debridement and MGX in each patient are shown in Fig. 2. In additional analysis, MMP-9 positivity rate significantly decreased $(P=0.014)$ at 4 weeks after the treatment (12 out of 24 cases; $50.0 \%$ ) compared to pre-treatment (20 out of 24 cases; $83.3 \%$ ). There was no allergic reaction and mechanical erosion observed post-treatment.

\section{Discussion}

In the present study, we demonstrated that combined treatment of lid debris debridement using the BlephEx and MGX significantly improved clinical findings, subjective symptoms, meibomian gland functions, and MMP-9 level in patients with moderate to severe MGD, advocating the use of this treatment combination as an effective strategy in treatment of moderate to severe MGD.

Eyelid debris debridement using the BlephEx was developed in the belief that the bacterial biofilm along the eyelid margin is responsible for meibomian gland inflammation [28]. Nattis et al. suggested that bacteria colonize the eyelid margin within a structure known as a biofilm, which causes eyelid inflammation, leading to MGD and aqueous insufficiency. Untreated MGD will inevitably result in eyelid destruction [29]. Normal bacterial floras, which are mainly Staphylococcus epidermidis and Staphylococcus aureus, living in the conjunctival sac and eyelid margin produce a biofilm that accumulates over many years [30]. When the population of Staphylococcus reaches a certain density, the quorum-sensing genes are activated by chemical signaling between organisms using homo-serine lactones and ion channels [31]. In turn, this gene activation results in the production of toxins and virulence factors that attack the host tissue to facilitate the expansion of the over-crowded biofilm [32,33]. Based on those reports suggesting the role of biofilm as a virulence factor in the ocular surface inflammation, routine mechanical removal of the biofilm on the eyelid margin cannot be more emphasized in prevention of development and aggravation of ocular surface inflammation. Consequently, the use of BlephEx, a convenient tool for eyelid debris debridement, was introduced and has been applied in clinical settings as an effective way to treat eyelid and ocular surface inflammation [18].

Table 1 Clinical signs and subjective symptoms before and after eyelid debris debridement and meibomian gland expression in patients with moderate and severe meibomian gland dysfunction

\begin{tabular}{|c|c|c|c|}
\hline Parameters & Before treatment & 4 weeks after the treatment & $P$ value \\
\hline$\overline{\text { TBUT }(\sec )^{a}}$ & $4.21(0.30)$ & $5.55(0.21)$ & 0.002 \\
\hline \multicolumn{4}{|l|}{ Fluorescein staining } \\
\hline SICCA staining score ${ }^{a}$ & $4.72(0.25)$ & $3.17(0.22)$ & $<0.001$ \\
\hline Oxford staining score ${ }^{a}$ & $1.67(0.10)$ & $1.15(0.09)$ & $<0.001$ \\
\hline Schirmer test $(\mathrm{mm})^{a}$ & $10.65(1.32)$ & $11.12(1.49)$ & 0.703 \\
\hline \multicolumn{4}{|l|}{ Subjective symptom } \\
\hline OSDI score ${ }^{a}$ & 55.79 (3.18) & $45.27(2.98)$ & 0.002 \\
\hline Ocular irritation symptom score ${ }^{b}$ & $33(73.3 \%)$ & $24(53.3 \%)$ & $<0.001$ \\
\hline
\end{tabular}

TBUT tear film break-up time, SICCA Sjögren's International Collaborative Clinical Alliance, OSDI ocular surface disease index

${ }^{a}$ Results are presented as mean (standard error) and $P$ values are from linear mixed model

${ }^{\mathrm{b}}$ Generalized estimating equations model for noncontinuous scale values: ocular irritation symptom score, $\mathrm{n}(\%$, proportion $\geq$ grade 2 ) 
Table 2 Lid margin abnormality, meibomian gland function, and meibomian gland dysfunction stage before and after eyelid debris debridement and meibomian gland expression in patients with moderate and severe meibomian gland dysfunction

\begin{tabular}{|c|c|c|c|}
\hline Parameters & Before treatment & 4 weeks after the treatment & $P$ value \\
\hline \multicolumn{4}{|l|}{ Lid margin abnormality } \\
\hline \multicolumn{4}{|l|}{ Lid margin irregularity $^{a}$} \\
\hline UL (0-2) & $1.57(0.07)$ & $1.32(0.08)$ & 0.047 \\
\hline $\mathrm{LL}(0-2)$ & $1.57(0.07)$ & $1.36(0.09)$ & 0.117 \\
\hline \multicolumn{4}{|l|}{ Telangiectasia $^{a}$} \\
\hline UL (0-3) & $1.96(0.07)$ & $1.37(0.10)$ & $<0.001$ \\
\hline $\mathrm{LL}(0-3)$ & $1.96(0.07)$ & $1.43(0.10)$ & $<0.001$ \\
\hline \multicolumn{4}{|l|}{ Meibomian gland orifice plugging ${ }^{a}$} \\
\hline UL (0-2) & $1.79(0.07)$ & $1.05(0.09)$ & $<0.001$ \\
\hline $\mathrm{LL}(0-2)$ & $1.75(0.09)$ & $1.09(0.09)$ & $<0.001$ \\
\hline \multicolumn{4}{|l|}{ Thickness $^{\mathrm{a}}$} \\
\hline UL (0-2) & $1.60(0.07)$ & $1.02(0.08)$ & $<0.001$ \\
\hline $\mathrm{LL}(0-2)$ & $1.54(0.07)$ & $1.07(0.09)$ & $<0.001$ \\
\hline Mucocutaneous junction replacement ${ }^{\mathrm{b}}$ & $26(56.5 \%)$ & $18(40.9 \%)$ & 0.115 \\
\hline \multicolumn{4}{|l|}{ Meibum characteristics } \\
\hline \multicolumn{4}{|l|}{ Meibum color ${ }^{a}$} \\
\hline UL (1-3) & $1.92(0.07)$ & $1.66(0.09)$ & 0.026 \\
\hline $\mathrm{LL}(1-3)$ & $1.96(0.09)$ & $1.48(0.08)$ & $<0.001$ \\
\hline \multicolumn{4}{|l|}{ Meibum consistency $^{\mathrm{a}}$} \\
\hline UL (1-3) & $1.69(0.09)$ & $1.25(0.07)$ & $<0.001$ \\
\hline LL (1-3) & $1.65(0.07)$ & $1.23(0.06)$ & $<0.001$ \\
\hline \multicolumn{4}{|l|}{ Meibum grade ${ }^{b}$} \\
\hline UL (0-3) & 38 (79.2\%) & $12(25.0 \%)$ & $<0.001$ \\
\hline MGD stage ${ }^{b}$ & 39 (84.8\%) & $10(21.7 \%)$ & $<0.001$ \\
\hline
\end{tabular}

MGD meibomian gland dysfunction, UL upper lid, LL lower lid

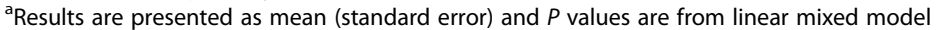

${ }^{\mathrm{b}}$ Generalized estimating equations model for noncontinuous scale values: mucocutaneous junction replacement, $\mathrm{n}$ (\%, proportion for positive result), meibum grade, $\mathrm{n}$ (\%, proportion $\geq$ grade 2$)$, MGD stage, $\mathrm{n}(\%$, proportion $\geq$ stage 3 )

In our study, eyelid debris debridement combined with MGX yielded a significant improvement in TBUT, SICCA ocular staining score, Oxford staining score, MGD stage, and OSDI questionnaire score at 4 weeks after treatment. In one recent study evaluating the effect of Cliradex terpinen-4-ol medicated lid scrubs and micro-blepharoexfoliation using the BlephEx for the treatment of Demodex blepharitis, this combination of treatment showed a statistically significant reduction in D. folliculorum infestation levels [18]. However, there was no significant improvement in OSDI score, tear osmolarity, extracellular MMP-9 levels, Schirmer 1 test, lid margin appearance, meibomian gland dropout, and meibomian gland secretions. Previous attempts to treat blepharitis associated with $D$. folliculorum include tea tree oil lid scrubs, oral and topical ivermectin, and oral metronidazole, but with varying reported success rates $[34,35]$. Additionally, demodex blepharitis is usually accompanied by meibomian gland dropout and cicatricial eyelid remodeling, thus making treatment more difficult. In our study, based upon relatively poor response of micro-blepharoexfoliation using the BlephEx in the treatment of Demodex blepharitis, we aimed to evaluate the effects of eyelid debris debridement combined with MGX in the treatment of moderate and severe MGD.

We demonstrated a significant improvement in the mean value of MMP-9 levels $(P=0.029)$ and proportion of patients showing positive result for MMP-9 levels $(P=0.014)$ at 4 weeks after the treatment $(50.0 \%$ after treatment versus $83.3 \%$ before treatment). This finding is consistent with previous study, which showed that an improvement in meibum expressibility positively correlated with the reduction of the tear inflammatory cytokines [36]. Ocular surface inflammation stimulates MMP-9 production by corneal epithelium and fibroblasts. Elevation of MMP-9 level on ocular surface in turn escalates production of other inflammatory cytokines and mediators exacerbating the chronic 


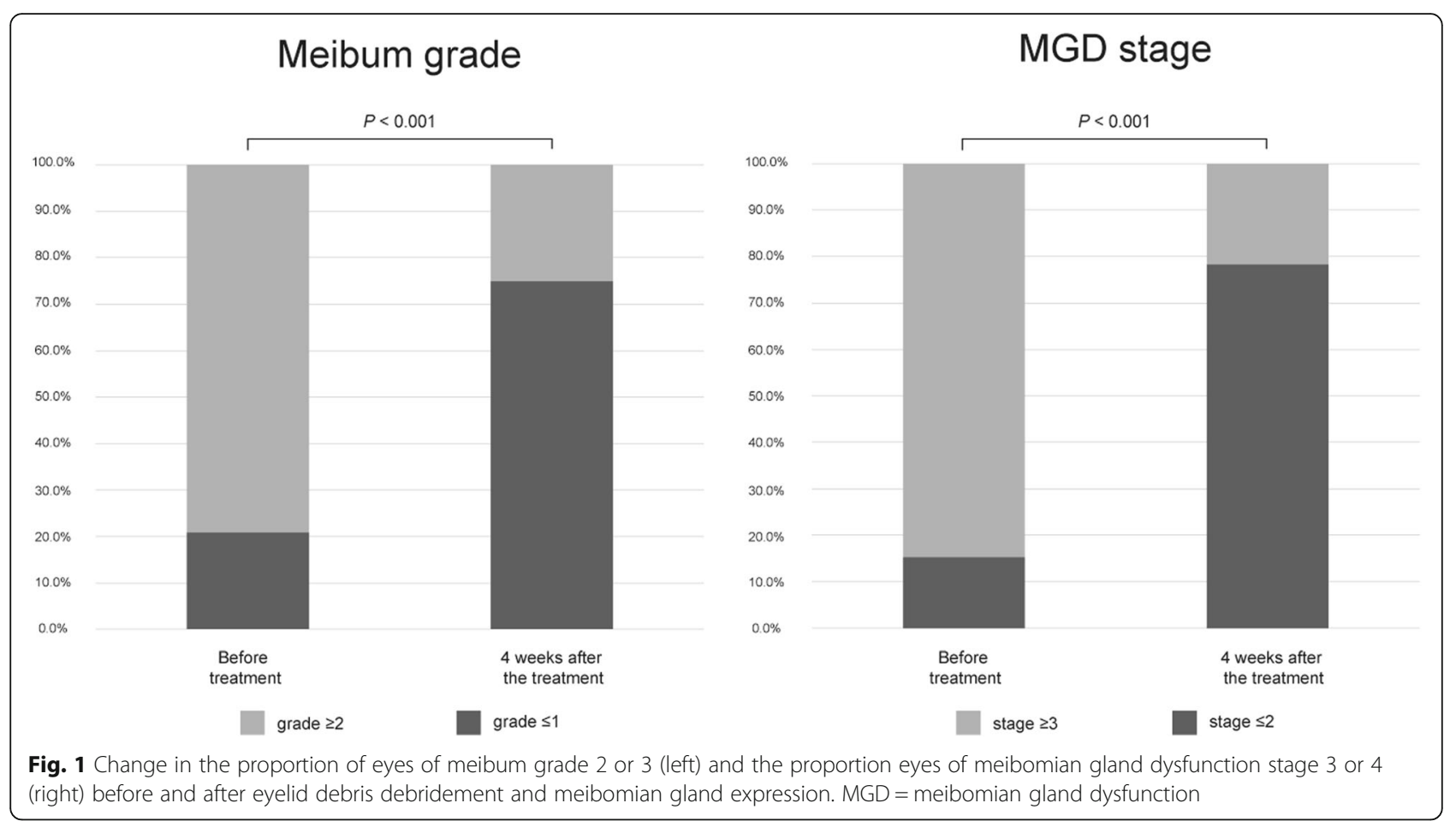

inflammatory cycle [37]. Elevation of MMP-9 level is associated with poor epithelial healing, which is also responsible for ocular surface inflammation and dry eye $[25,38]$. As demonstrated previously, tear film MMP-9 level is a reliable quantitative index for ocular surface inflammation and tends to increase proportionally with the severity of dry eye. Its activity was significantly elevated in patients with MGD as well $[39,40]$. MMP-9 immunoassay device is a rapid and precise tool that can measure MMP-9 in tear film with a sensitivity of $85 \%$, a specificity of $94 \%$, a negative predictive value of $73 \%$, and a positive predictive value of $97 \%$ in diagnosing dry eye $[25,41]$. We evaluated the correlation between MMP-9 level and clinical findings using Spearman rankorder correlation which showed a statistically significant association. Increase in MMP-9 level was associated with decrease in TBUT $\left(P=0.026, R^{2}=-0.338\right)$, increase in SICCA staining score $\left(P=0.001, R^{2}=0.451\right)$, increase in
Oxford staining score $\left(P=0.001, R^{2}=0.497\right)$, and exacerbation of MGD grading $\left(P=0.009, R^{2}=0.468\right)$ [42]

Meibum characteristic also showed statistically significant improvement after use of BlephEx followed by MGX. One possible explanation for this would be the removal of biofilm which subsequently reduces lipaseproducing bacterial burden on the eyelid [43]. Bacterial lipase can affect the composition of meibum, which in turn can change the composition of the lipid layer. This disruption of homeostasis results in tear film instability and ocular surface inflammation [28, 30, 44, 45]. The blockage of meibomian gland ducts further induces bacterial proliferation, which facilitates the production of more lipase and finally induces a vicious cycle [46-48].

Lid margin abnormalities such as telangiectasia, meibomian gland plugging and thickness significantly improved at 4 weeks after the treatment. However, lid margin irregularity and mucocutaneous junction

Table 3 Changes in matrix metalloproteinase-9 levels before and after eyelid debris debridement and meibomian gland expression in patients with moderate and severe meibomian gland dysfunction

\begin{tabular}{llll}
\hline Parameters & Before treatment & 4 weeks after the treatment & $P$ value \\
\hline MMP-9 levels $^{\mathrm{a}}$ & $2.58(0.20)$ & $1.92(0.26)$ & 0.029 \\
MMP-9 positivity $^{\mathrm{b}}$ & $20(83.3 \%)$ & $12(50.0 \%)$ & 0.014 \\
\hline
\end{tabular}

MMP-9 matrix metalloproteinase-9

MMP-9 levels were measured based upon the changes in the intensity of color on a scale of 0 to 4 , as follows: 0 , none; 1 , trace; 2 , weak positive; 3 , positive; and 4 , strong positive

${ }^{a}$ Results are presented as mean (standard error) and $P$ values are from linear mixed model

${ }^{b} X^{2}$ test: $n$ (\%, proportion for positive result: weak, positive, and strong positive) 


\section{Changes in MMP-9 levels}

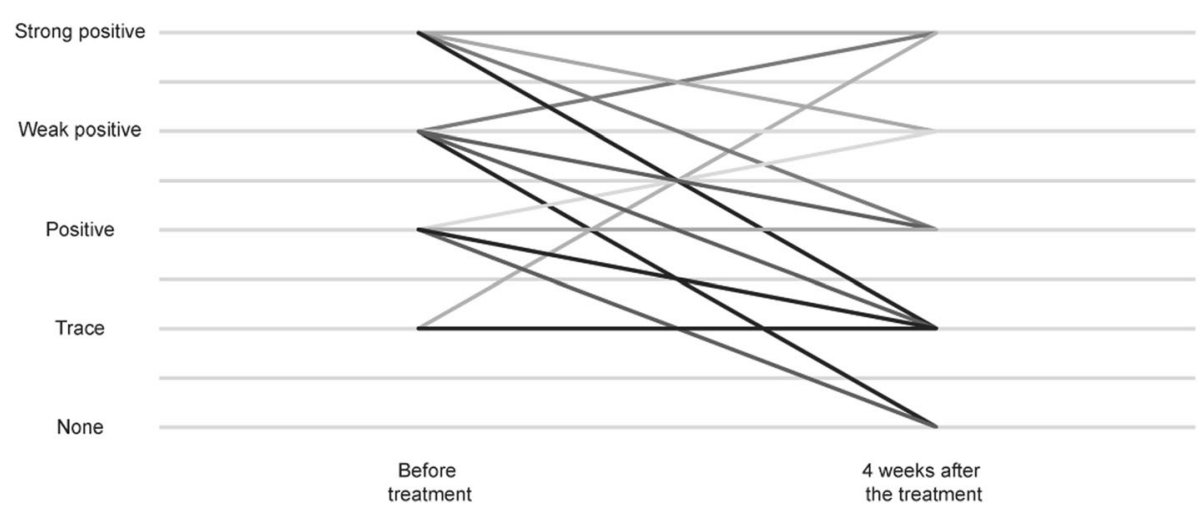

Fig. 2 Changes in matrix metalloproteinase-9 levels before and after eyelid debris debridement and meibomian gland expression in each patient. MMP-9 = matrix metalloproteinase-9

replacement showed no significant improvement. Lid margin telangiectasia, which is associated with vascular dilatation due to inflammatory mediators, and meibomian gland orifice plugging, which is related to altered meibum composition, are two features of dry dye disease that are relatively easier to reverse [46]. However, lid margin irregularity and mucocutaneous junction replacement, which develop over time due to chronic inflammation, are structural changes that take more time and are difficult to reverse into a normal structure [1, 19, 48]. Thus, it would only be fair to predict that more than one session of lid scrubbing with the BlephEx is required in order to detect any positive change in eyelid margin irregularity and mucocutaneous junction. As this study evaluated the effect of one session of lid debris debridement and MGX in treating moderate and severe MGD, a further study to assess the efficacy and safety of extended session of lid debris debridement and MGX seems necessary.

This study is limited by a small number of patients and its retrospective nature. Secondly, the duration of follow-up was limited to 4 weeks after treatment. Given that the follow-up period after one session of treatment was short, further investigation is needed to assess the long-term effectiveness and safety of multiple rounds of lid debridement. And prospective randomized controlled study with proper sample size is also necessary. It would also be helpful to evaluate the change of additional tear cytokines and composition of meibum profile after eyelid margin debridement in order to expand our understanding in the mechanism of dry eye disease.

\section{Conclusions}

We have shown that one session of combination of eyelid debris debridement using the BlephEx and MGX is an effective treatment modality for patients with moderate and severe MGD in terms of improving patient symptoms and clinical signs, and reducing level of MMP-9 in treated patients. We believe that the improvement in signs and symptoms could be attributed to decreased MMP-9 level detected after treatment. Further study is mandatory to evaluate the efficacy and safety of extended session of lid debris debridement and MGX.

Acknowledgements

We would like to thank Editage (www.editage.co.kr) for English language editing.

\section{Authors' contributions}

Study concept and design (SYM, SAH, HJK, SYP, JHL, HSC, JYK, HT, HL); collection, management, analysis, and interpretation of data (SYM, SAH, HJK, SYP, JHL, HSC, JYK, HT, HL); and preparation, review, or approval of the manuscript (SYM, SAH, JYK, HT, HL). All authors read and approved the final manuscript.

\section{Funding}

This work was supported by the Korea Medical Device Development Fund grant funded by the Korea government (the Ministry of Science and ICT, the Ministry of Trade, Industry and Energy, the Ministry of Health \& Welfare, the Ministry of Food and Drug Safety) (Project Number: 9991006821 ,

KMDF_PR_20200901_0148) and by a grant from the Asan Institute for Life Sciences, Asan Medical Center, Seoul, Korea (2021IP0059-1, 2021IP0061-1). The funder had no role in the design and conduct of the study; collection, management, analysis, and interpretation of the data; preparation, review, or approval of the manuscript; and decision to submit the manuscript for publication.

Availability of data and materials

Data are available upon request from the authors.

\section{Declarations}

\section{Ethics approval and consent to participate}

This study was conducted with the approval of the Institutional Review Board of the Asan Medical Center and the University of Ulsan College of Medicine, Seoul, South Korea (2020-0096).

Consent for publication

Not applicable. 


\section{Competing interests}

The authors have no proprietary or financial interest in the materials presented herein.

Received: 15 September 2020 Accepted: 29 March 2021

Published online: 12 April 2021

\section{References}

1. Craig JP, Nichols KK, Akpek EK, Caffery B, Dua HS, Joo CK, et al. TFOS DEWS II definition and classification report. Ocul Surf. 2017;15(3):276-83. https:// doi.org/10.1016/j.jtos.2017.05.008.

2. Alghamdi YA, Mercado C, McClellan AL, Batawi H, Karp CL, Galor A. Epidemiology of meibomian gland dysfunction in an elderly population. Cornea. 2016;35(6):731-5. https://doi.org/10.1097//CO.0000000000000815.

3. Lemp MA, Crews LA, Bron AJ, Foulks GN, Sullivan BD. Distribution of aqueous-deficient and evaporative dry eye in a clinic-based patient cohort: a retrospective study. Cornea. 2012;31(5):472-8. https://doi.org/10.1097/ICO. Ob013e318225415a.

4. Mathers WD. Ocular evaporation in meibomian gland dysfunction and dry eye. Ophthalmology. 1993;100(3):347-51. https://doi.org/10.1016/S0161-642 O(93)31643-X.

5. Nelson JD, Shimazaki J, Benitez-del-Castillo JM, Craig JP, McCulley JP, Den S, et al. The international workshop on meibomian gland dysfunction: report of the definition and classification subcommittee. Invest Ophthalmol Vis Sci. 2011;52(4):1930-7. https://doi.org/10.1167/iovs.10-6997b.

6. Schaumberg DA, Uchino M, Christen WG, Semba RD, Buring JE, Li JZ. Patient reported differences in dry eye disease between men and women: impact, management, and patient satisfaction. PLoS One. 2013;8(9):e76121. https://doi.org/10.1371/journal.pone.0076121.

7. Shen Lee B, Kabat AG, Bacharach J, Karpecki P, Luchs J. Managing dry eye disease and facilitating realistic patient expectations: a review and appraisal of current therapies. Clin Ophthalmol. 2020;14:119-26. https://doi.org/10.214 7/OPTH.S228838

8. Lee H, Chung B, Kim KS, Seo KY, Choi BJ, Kim TI. Effects of topical loteprednol etabonate on tear cytokines and clinical outcomes in moderate and severe meibomian gland dysfunction: randomized clinical trial. Am J Ophthalmol. 2014;158(6):1172-1183 e1171. https://doi.org/10.1016/j.ajo.2014. 08.015 .

9. Ciloglu E, Ozcan AA, Incekalan T, Unal F. The role of topical azithromycin in the treatment of meibomian gland dysfunction. Cornea. 2020;39(3):321-4. https://doi.org/10.1097//CO.0000000000002233.

10. Lee H, Min K, Kim EK, Kim TI. Minocycline controls clinical outcomes and inflammatory cytokines in moderate and severe meibomian gland dysfunction. Am J Ophthalmol. 2012;154(6):949-957 e941. https://doi.org/1 0.1016/j.ajo.2012.06.009.

11. Geerling G, Tauber J, Baudouin C, Goto E, Matsumoto Y, O'Brien T, et al. The international workshop on meibomian gland dysfunction: report of the subcommittee on management and treatment of meibomian gland dysfunction. Invest Ophthalmol Vis Sci. 2011;52(4):2050-64. https://doi.org/1 $0.1167 /$ iovs.10-6997g

12. Xue AL, Wang MTM, Ormonde SE, Craig JP. Randomised double-masked placebo-controlled trial of the cumulative treatment efficacy profile of intense pulsed light therapy for meibomian gland dysfunction. Ocul Surf. 2020.

13. Piyacomn $Y$, Kasetsuwan N, Reinprayoon U, Satitpitakul V, Tesapirat L. Efficacy and safety of intense pulsed light in patients with meibomian gland dysfunction-a randomized, double-masked, sham-controlled clinical trial. Cornea. 2020;39(3):325-32. https://doi.org/10.1097/ICO.0000000000002204.

14. Sabeti S, Kheirkhah A, Yin J, Dana R. Management of meibomian gland dysfunction: a review. Surv Ophthalmol. 2020;65(2):205-17. https://doi.org/1 0.1016/j.survophthal.2019.08.007.

15. Arita R, Fukuoka S, Morishige N. Therapeutic efficacy of intense pulsed light in patients with refractory meibomian gland dysfunction. Ocul Surf. 2019; 17(1):104-10. https://doi.org/10.1016/j.jtos.2018.11.004.

16. Arita R, Mizoguchi T, Fukuoka S, Morishige N. Multicenter study of intense pulsed light therapy for patients with refractory meibomian gland dysfunction. Cornea. 2018;37(12):1566-71. https://doi.org/10.1097/ICO. 0000000000001687.

17. Sambhi RS, Sambhi GDS, Mather R, Malvankar-Mehta MS. Intense pulsed light therapy with meibomian gland expression for dry eye disease. Can J Ophthalmol. 2020;55(3):189-98. https://doi.org/10.1016/j.jcjo.2019.11.009.
18. Epstein IJ, Rosenberg E, Stuber R, Choi MB, Donnenfeld ED, Perry HD. Double-masked and unmasked prospective study of terpinen-4-ol lid scrubs with microblepharoexfoliation for the treatment of demodex blepharitis. Cornea. 2020;39(4):408-16. https://doi.org/10.1097/ICO.0000000000002243.

19. Nichols KK, Foulks GN, Bron AJ, Glasgow BJ, Dogru M, Tsubota K, et al. The international workshop on meibomian gland dysfunction: executive summary. Invest Ophthalmol Vis Sci. 2011;52(4):1922-9. https://doi.org/10.11 67/iovs.10-6997a.

20. Tomlinson A, Bron AJ, Korb DR, Amano S, Paugh JR, Pearce El, et al. The international workshop on meibomian gland dysfunction: report of the diagnosis subcommittee. Invest Ophthalmol Vis Sci. 2011;52(4):2006-49. https://doi.org/10.1167/iovs.10-6997f.

21. Fukuoka S, Arita R. Tear film lipid layer increase after diquafosol instillation in dry eye patients with meibomian gland dysfunction: a randomized clinical study. Sci Rep. 2019;9(1):9091. https://doi.org/10.1038/s41598-019-4 5475-7.

22. Rasmussen A, Ice JA, Li H, Grundahl K, Kelly JA, Radfar L, et al. Comparison of the American-European Consensus Group Sjogren's syndrome classification criteria to newly proposed American College of Rheumatology criteria in a large, carefully characterised sicca cohort. Ann Rheum Dis. 2014; 73(1):31-8. https://doi.org/10.1136/annrheumdis-2013-203845.

23. Whitcher JP, Shiboski CH, Shiboski SC, Heidenreich AM, Kitagawa K, Zhang $\mathrm{S}$, et al. A simplified quantitative method for assessing keratoconjunctivitis sicca from the Sjogren's Syndrome International Registry. Am J Ophthalmol. 2010;149(3):405-15. https://doi.org/10.1016/j.ajo.2009.09.013.

24. Lee H, Han YE, Park SY, Lee JH, Chung HS, Moon SY, et al. Changes in the expression of matrix metalloproteinase- 9 after intense pulsed light therapy combined with meibomian gland expression in moderate and severe meibomian gland dysfunction. Cont Lens Anterior Eye. 2020. https://doi. org/10.1016/j.clae.2020.05.008.

25. Chotikavanich S, de Paiva CS, de Li Q, Chen JJ, Bian F, Farley WJ, et al. Production and activity of matrix metalloproteinase-9 on the ocular surface increase in dysfunctional tear syndrome. Invest Ophthalmol Vis Sci. 2009; 50(7):3203-9. https://doi.org/10.1167/iovs.08-2476.

26. Shimazaki J, Sakata M, Tsubota K. Ocular surface changes and discomfort in patients with meibomian gland dysfunction. Arch Ophthalmol. 1995;113(10): 1266-70. https://doi.org/10.1001/archopht.1995.01100100054027.

27. Korb DR, Blackie CA. Meibomian gland therapeutic expression: quantifying the applied pressure and the limitation of resulting pain. Eye Contact Lens. 2011;37(5):298-301. https://doi.org/10.1097//CL.0b013e31821bc7c5.

28. Rynerson JM, Perry HD. DEBS - a unification theory for dry eye and blepharitis. Clin Ophthalmol. 2016;10:2455-67. https://doi.org/10.2147/OPTH. S114674.

29. Nattis A, Perry HD, Rosenberg ED, Donnenfeld ED. Influence of bacterial burden on meibomian gland dysfunction and ocular surface disease. Clin Ophthalmol. 2019;13:1225-34. https://doi.org/10.2147/OPTH.S215071.

30. Dong $X$, Wang $Y$, Wang $W$, Lin $P$, Huang Y. Composition and diversity of bacterial community on the ocular surface of patients with meibomian gland dysfunction. Invest Ophthalmol Vis Sci. 2019;60(14):4774-83. https:// doi.org/10.1167/iovs.19-27719.

31. Mireille Aye A, Bonnin-Jusserand M, Brian-Jaisson F, Ortalo-Magne A, Culioli $G$, Koffi Nevry R, et al. Modulation of violacein production and phenotypes associated with biofilm by exogenous quorum sensing $\mathrm{N}$-acylhomoserine lactones in the marine bacterium Pseudoalteromonas ulvae TC14. Microbiology. 2015;161(10):2039-51. https://doi.org/10.1099/mic.0.000147.

32. Hastings JW, Greenberg EP. Quorum sensing: the explanation of a curious phenomenon reveals a common characteristic of bacteria. J Bacteriol. 1999; 181(9):2667-8. https://doi.org/10.1128/JB.181.9.2667-2668.1999.

33. Yarwood JM, Schlievert PM. Quorum sensing in Staphylococcus infections. J Clin Invest. 2003;112(11):1620-5. https://doi.org/10.1172/JCI200320442.

34. Salem DA, El-Shazly A, Nabih N, El-Bayoumy Y, Saleh S. Evaluation of the efficacy of oral ivermectin in comparison with ivermectin-metronidazole combined therapy in the treatment of ocular and skin lesions of Demodex folliculorum. Int J Infect Dis. 2013;17(5):e343-7. https://doi.org/10.1016/j.jijid.2 012.11.022.

35. Gao YY, Di Pascuale MA, Elizondo A, Tseng SC. Clinical treatment of ocular demodecosis by lid scrub with tea tree oil. Cornea. 2007;26(2):136-43. https://doi.org/10.1097/01.ico.0000244870.62384.79.

36. Choi M, Han SJ, Ji YW, Choi YJ, Jun I, Alotaibi MH, et al. Meibum expressibility improvement as a therapeutic target of intense pulsed light treatment in meibomian gland dysfunction and its association with tear 
inflammatory cytokines. Sci Rep. 2019;9(1):7648. https://doi.org/10.1038/s41 598-019-44000-0.

37. Afonso AA, Sobrin L, Monroy DC, Selzer M, Lokeshwar B, Pflugfelder SC. Tear fluid gelatinase $B$ activity correlates with IL-1alpha concentration and fluorescein clearance in ocular rosacea. Invest Ophthalmol Vis Sci. 1999; 40(11):2506-12.

38. Kaufman HE. The practical detection of MMP-9 diagnoses ocular surface disease and may help prevent its complications. Cornea. 2013;32(2):211-6. https://doi.org/10.1097//CO.0b013e3182541e9a.

39. Solomon A, Dursun D, Liu Z, Xie Y, Macri A, Pflugfelder SC. Pro- and antiinflammatory forms of interleukin-1 in the tear fluid and conjunctiva of patients with dry-eye disease. Invest Ophthalmol Vis Sci. 2001;42(10):228392.

40. Sambursky R, Davitt WF 3rd, Latkany R, Tauber S, Starr C, Friedberg M, et al. Sensitivity and specificity of a point-of-care matrix metalloproteinase 9 immunoassay for diagnosing inflammation related to dry eye. JAMA Ophthalmol. 2013;131(1):24-8. https://doi.org/10.1001/jamaophthalmol.2013. 561.

41. Sambursky R, Davitt WF 3rd, Friedberg M, Tauber S. Prospective, multicenter, clinical evaluation of point-of-care matrix metalloproteinase-9 test for confirming dry eye disease. Cornea. 2014;33(8):812-8. https://doi.org/10.1 097/ICO.0000000000000175.

42. VanDerMeid KR, Su SP, Ward KW, Zhang JZ. Correlation of tear inflammatory cytokines and matrix metalloproteinases with four dry eye diagnostic tests. Invest Ophthalmol Vis Sci. 2012;53(3):1512-8. https://doi.org/10.1167/iovs.117627.

43. Flanagan JL, Khandekar N, Zhu H, Watanabe K, Markoulli M, Flanagan JT, et al. Glycerol monolaurate inhibits lipase production by clinical ocular isolates without affecting bacterial cell viability. Invest Ophthalmol Vis Sci. 2016;57(2):544-50. https://doi.org/10.1167/iovs.15-17180.

44. Souchier M, Joffre C, Gregoire S, Bretillon L, Muselier A, Acar N, et al. Changes in meibomian fatty acids and clinical signs in patients with meibomian gland dysfunction after minocycline treatment. Br J Ophthalmol. 2008;92(6):819-22. https://doi.org/10.1136/bjo.2007.133900.

45. Borchman D, Ramakrishnan V, Henry C, Ramasubramanian A. Differences in meibum and tear lipid composition and conformation. Cornea. 2020;39(1): 122-8. https://doi.org/10.1097/ICO.0000000000002095.

46. Knop E, Knop N, Millar T, Obata H, Sullivan DA. The international workshop on meibomian gland dysfunction: report of the subcommittee on anatomy, physiology, and pathophysiology of the meibomian gland. Invest Ophthalmol Vis Sci. 2011;52(4):1938-78. https://doi.org/10.1167/iovs.106997c.

47. Xiao J, Adil MY, Chen X, Utheim OA, Raeder S, Tonseth KA, et al. Functional and morphological evaluation of meibomian glands in the assessment of meibomian gland dysfunction subtype and severity. Am J Ophthalmol. 2020;209:160-7. https://doi.org/10.1016/j.ajo.2019.09.005.

48. Baudouin C, Messmer EM, Aragona P, Geerling G, Akova YA, Benitez-delCastillo J, et al. Revisiting the vicious circle of dry eye disease: a focus on the pathophysiology of meibomian gland dysfunction. Br J Ophthalmol. 2016;100(3):300-6. https://doi.org/10.1136/bjophthalmol-2015-307415.

\section{Publisher's Note}

Springer Nature remains neutral with regard to jurisdictional claims in published maps and institutional affiliations.

Ready to submit your research? Choose BMC and benefit from:
- fast, convenient online submission
- thorough peer review by experienced researchers in your field
- rapid publication on acceptance
- support for research data, including large and complex data types
- gold Open Access which fosters wider collaboration and increased citations
- maximum visibility for your research: over 100M website views per year
At BMC, research is always in progress.
Learn more biomedcentral.com/submissions

